\title{
Stability Analysis of Trilateral Haptic Collaboration
}

\author{
Jian $\mathrm{Li}^{*} \quad$ Mahdi Tavakoli ${ }^{\dagger} \quad$ Qi Huang ${ }^{\ddagger}$ \\ $* \vdots$ School of Energy Science and Engineering, University of Electronic Science and Technology of China, \\ Chengdu, Sichuan, China 611731 \\ ${ }^{* \dagger}$ Department of Electrical and Computer Engineering, University of Alberta, Edmonton, AB T6G 2V4, Canada
}

\begin{abstract}
This paper presents a criterion for absolute stability of a general class of three-port networks. Trilateral haptic systems, which have recently found many interesting applications, can be modeled as three-port networks. Traditionally, existing criteria (Llewellyn's criterion) have facilitated the stability analysis of bilateral haptic systems modeled as two-port networks. If the same criteria were to be used for stability analysis of a three-port network, its third port would need to be assumed known for it to reduce to a two-port network. However, this is restrictive because, according to the definition of absolute stability, all three terminations of the three-port network must be allowed to be arbitrary (while passive).

In this paper, extending Llewellyn's criterion, we present closedform necessary and sufficient conditions for absolute stability of a general class of three-port networks - the three terminations need to be passive but are otherwise arbitrary. To this end, we first find a symmetrization condition under which a general asymmetric impedance (or admittance) matrix $Z_{3 \times 3}$ has an equivalent symmetric counterpart $Z_{e q}$; this $Z_{e q}$ models a reciprocal three-port network with the same stability characterization as the general nonreciprocal three-port network modeled by $Z$. Then, based on the equivalence of passivity and absolute stability for the equivalent reciprocal network, an absolute stability condition for the original nonreciprocal network is derived. To show how the resulting absolute stability criterion can be utilized at the system design stage, we have applied it to the problem of designing controllers for triple-user collaborative haptic virtual environment systems. The validity of the resulting absolute stability conditions have been verified via simulations.
\end{abstract}

Index Terms: H.5.2 [Information Interfaces and Presentation]: User Interfaces-Haptic I/O; I.2.9 [Artificial Intelligence]: Problem Solving, Control Methods, and Search—Control theory

\section{INTRODUCTION}

For coupled stability analysis of bilateral teleoperation system, the human operator's and the environment's dynamics and the teleoperator immitance $(z, y, h$, and $g)$ parameters are needed. Here, the teleoperator comprises the master, the slave, their controllers, and the communication channel. In practice, the models of the human operator and the environment can be unknown, uncertain, and/or time-varying. Thus, absolute or unconditional stability of a bilateral teleoperator assuming that the human operator and the environment demonstrate passive behaviors is analyzed via Llewellyn's stability criterion for two-port networks [1, 2, 3]. For brevity, absolute or unconditional stability is simply referred to as "stability" in the rest of the paper. "Coupled stability" will refer to BIBO stability of a network when it is coupled to terminations at all if its ports.

*e-mail: jian1@ualberta.ca

†e-mail:mahdi.tavakoli@ualberta.ca

†e-mail:huangqi@uestc.edu.cn

IEEE World Haptics Conference 2013

14-18 April, Daejeon, Korea

978-1-4799-0088-6/13/\$31.00 @2013 IEEE
Recently, new application scenarios have emerged that involve the collaboration of multiple users in teleoperation of a robot or in performing a haptic virtual task. Examples of these new applications are tele-rehabilitation [4], surgical training, [5], and cooperative multi-robot systems[6]. Specifically, dual-user teleoperation of a robot and triple-user collaborative haptic virtual environments have given rise to trilateral haptic systems. A difference between a trilateral and a bilateral haptic system is that they are modeled as a three-port and a two-port network, respectively. Thus, conventional theories for stability analysis of bilateral haptic systems will not be adequate for trilateral haptic systems.

In contrast to the stability criteria for two-port networks, which have only involved conditions on the immitance parameters of the two-port network and are independent of the port terminations, past research has been struggling to find a similar stability condition for three-port networks independent of the port terminations. Instead, in past research $[7,8,9]$, the third port was assumed to be coupled to a known termination such that the three-port network reduced to a two-port network, paving the way for the application of Llewellyn's criterion. The limiting factor of this approach is that the resulting stability condition will inevitably depend on the immitance of the third port's termination. This is restrictive because not allowing all three terminations of the three-port network to be arbitrary (while passive) contradicts the very definition of stability (again, throughout this paper, all references are to absolute or unconditional stability).

Using the aforementioned approach, namely, reducing a given three-port network to a two-port network by assuming a known termination for the third port, Boehm et al. in [10] established nine conditions for determining the stability of a three-port network described by its scattering $(S)$ parameters. The approach in [8] reduced a three-port network to three two-port networks by terminating each of the three ports, and managed to reduce the number of conditions from nine to three. Also, Kuo et al. [7] reduced a threeport network to a two-port network by coupling the third port to a known termination and then required the input reflection coefficients at the first and the second ports to be less than unity. Unfortunately, in the above approaches, a degree of freedom is lost when the third port is coupled to a known termination. Thus, there is a need for a tool that can directly analyze the stability of trilateral haptic systems modeled as three-port networks without reducing them to two-port networks. Such a tool, which will guarantee the coupled stability of the system under all passive but otherwise arbitrary terminations for all three ports, is developed in this paper.

Unlike past work, we would like to have a stability condition $d i$ rectly in the immittance (e.g., impedance $Z$ ) domain and not in the scattering $(S)$ domain. While the $S$-parameters are most accurately measured for higher-frequency systems such as microwave circuits, $Z$-parameters can be accurately measured in lower-frequency systems including robotic systems. In fact, the measurement of $Z$ parameters approaches zero in microwave circuits where the frequencies are very high (over $1 \mathrm{GHz}$ ), making the use of reflection coefficients and scattering parameters justifiable for the stability analysis. This explains the abundance of scattering parameters based stability conditions in the microwave systems literature (see, 
for example, [11]). Conversely, in robotic systems, the measurement of $S$-parameters is close to zero in any frequency range of practical interest and, therefore, it is highly desirable to have stability conditions that directly depend on the $Z$-parameters or other immittance parameters of the three-port network.

Inspired by $\mathrm{Ku}$ [12], who studied $\mathrm{N}$-port network stability when the impedance matrix of the network is of a tri-diagonal Jacobian form [12], in this paper we present a criterion to analyze the stability of a general class of nonreciprocal three-port networks. As a case study, we consider a triple-user collaborative haptic virtual environment system and use the proposed stability criterion to design stabilizing controllers for the system.

The rest of the paper is organized as follows: The next section reviews definitions of stability for general $N$-port networks and, for the special case of reciprocal networks, relates them to passivity. In Section 3, the proposed stability criterion for all nonreciprocal three-port networks that satisfy our so-called symmetrization condition is derived. Then, as a case study to show how the resulting stability criterion can be utilized, in Section 4, a triple-user collaborative haptic virtual environment system is considered and the stability conditions in terms of system parameters including controller gains are found. Finally, simulations to verify the validity of the calculated stability conditions for the triple-user collaborative haptic virtual environment system are presented in Section 5. Section 6 contains concluding remarks.

\section{Definitions and Criteria for N-Port Network STABILITY}

An $n$-port network is called stable if the port currents are zero under all passive terminations for all ports [13]. Similarly, an $n$-port network is called weakly stable if the port currents are zero under all strictly passive terminations for all ports. We know that an LTI termination is passive (strictly passive) if its impedance is nonnegative (positive) real [14]. Suppose the $n$-port network is terminated in arbitrary passive impedances $z_{1}, z_{2}, \cdots, z_{n}$, and the port currents at the respective ports are denoted by $I_{1}, I_{2}, \cdots, I_{n}$. Thus, it is immediately understood that a general $n$-port network with impedance matrix $Z_{n \times n}$ is stable (weakly stable) if and only if the equation

$$
\left(Z+Z_{0}\right) \mathbf{I}=0, \quad Z_{0}=\operatorname{diag}\left[z_{1}, z_{2}, \cdots, z_{n}\right]
$$

where $I=\left[I_{1}, I_{2}, \cdots, I_{n}\right]^{T}$ has only the trivial solution $\mathbf{I}=0$ for every choice of $n$ terminations $z_{1}, z_{2}, \cdots, z_{n}$ that are nonnegative (positive) real. In other words, the $n$-port network is stable (weakly stable) if and only if

$$
\operatorname{det}\left(Z+Z_{0}\right) \neq 0
$$

for any choice of $n$ terminations with nonnegative (positive) real parts. We remember that a nonnegative (positive) real impedance $z_{i}(j \omega)$ has a real part with a nonnegative (positive) value. A rational function $F(s)$ is positive real if and only if, in addition to being real for real $s, F(s)$ has no RHP poles, any poles of $F(s)$ on the imaginary axis are simple with real and non-negative residues, and $\operatorname{Re}[F(j \omega)] \geq 0, \forall \omega$.

There is an alternate definition for $n$-port network stability. Assume the input impedance (i.e., the driving-point impedances) at port $\kappa$ of an $n$-port network is $Z_{i n_{\kappa}}$ when all other $n-1$ ports are coupled to passive (strictly passive) terminations. Then, the $n$-port network is stable (weakly stable) if and only if

$$
\operatorname{Re}\left(Z_{i n_{\kappa}}\right)>0 \quad(\geq 0), \quad \kappa=1,2, \cdots, n
$$

for all passive (strictly passive) $z_{1}, z_{2}, \cdots, z_{n}$. Equivalently, the $n$-port network is stable (weakly stable) if and only if

$$
\int_{0}^{t} V_{\kappa}(\tau) I_{\kappa}(\tau) \mathrm{d} \tau>\quad(\geq 0), \quad \kappa=1,2, \cdots, n
$$

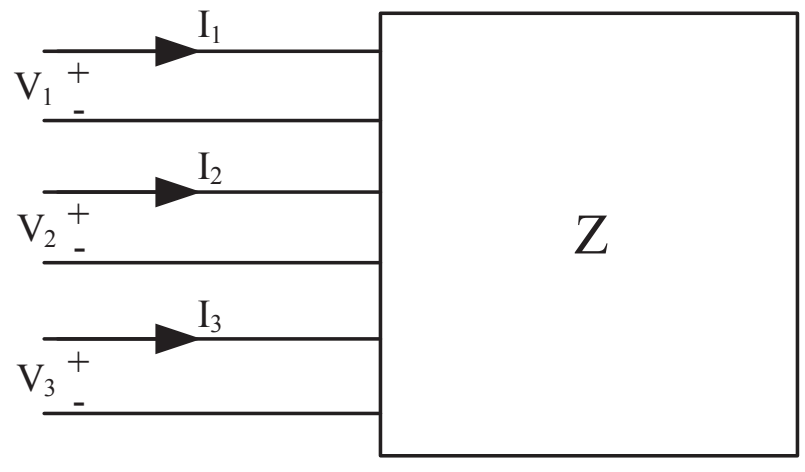

Figure 1: Three-port network

for all passive (strictly passive) $z_{1}, z_{2}, \cdots, z_{n}$ [15]. Conditions (3) or (4) represent an alternate way to examine the stability of $n$-port networks. In the following, the equivalence of (3) and (4) with the stability condition (2) is shown for $n=3$, i.e., a general nonreciprocal three-port network shown in Figure 1 with the impedance matrix

$$
Z=\left[\begin{array}{lll}
Z_{11} & Z_{12} & Z_{13} \\
Z_{21} & Z_{22} & Z_{23} \\
Z_{31} & Z_{32} & Z_{33}
\end{array}\right]
$$

For the three-port network (5), the input impedances at port 1 when ports 2 and 3 are terminated to $z_{2}$ and $z_{3}$, at port 2 when ports 1 and 3 are terminated to $z_{1}$ and $z_{3}$, and at port 3 when ports 1 and 2 are terminated to $z_{1}$ and $z_{2}$ are, respectively,

$$
\begin{aligned}
& Z_{i n_{1}}=Z_{11}-\frac{Z_{12} Z_{21}\left(Z_{33}+z_{3}\right)+Z_{13} Z_{31}\left(Z_{22}+z_{2}\right)-D}{\left(Z_{22}+z_{2}\right)\left(Z_{33}+z_{3}\right)-Z_{23} Z_{32}} \\
& Z_{i n_{2}}=Z_{22}-\frac{Z_{12} Z_{21}\left(Z_{33}+z_{3}\right)+Z_{23} Z_{32}\left(Z_{11}+z_{1}\right)-D}{\left(Z_{11}+z_{1}\right)\left(Z_{33}+z_{3}\right)-Z_{13} Z_{31}} \\
& Z_{i n_{3}}=Z_{33}-\frac{Z_{13} Z_{31}\left(Z_{22}+z_{2}\right)+Z_{23} Z_{32}\left(Z_{11}+z_{1}\right)-D}{\left(Z_{11}+z_{1}\right)\left(Z_{22}+z_{2}\right)-Z_{12} Z_{21}}
\end{aligned}
$$

where $D=Z_{13} Z_{21} Z_{32}-Z_{12} Z_{23} Z_{31}$. According to (2), for the nonreciprocal three-port network (5) to be stable (weakly stable), we must have

$$
\operatorname{det}\left(Z+Z_{0}\right) \neq 0, \quad Z_{0}=\operatorname{diag}\left[z_{1}, z_{2}, z_{3}\right]
$$

for any choice of passive (strictly passive) impedances $z_{1}, z_{2}$ and $z_{3}$. The stability condition (9) can be rewritten as

$$
\begin{aligned}
& \left(Z_{11}+z_{1}\right)\left(Z_{22}+z_{2}\right)\left(Z_{33}+z_{3}\right)-Z_{13} Z_{21} Z_{32}-Z_{12} Z_{23} Z_{31} \\
& \neq Z_{23} Z_{32}\left(Z_{11}+z_{1}\right)+Z_{12} Z_{21}\left(Z_{33}+z_{3}\right)+Z_{13} Z_{31}\left(Z_{22}+z_{2}\right)
\end{aligned}
$$

Now, if $\left(Z_{22}+z_{2}\right)\left(Z_{33}+z_{3}\right)-Z_{23} Z_{32} \neq 0$, (10) implies that

$$
-z_{1} \neq Z_{11}-\frac{Z_{12} Z_{21}\left(Z_{33}+z_{3}\right)+Z_{13} Z_{31}\left(Z_{22}+z_{2}\right)-D}{\left(Z_{22}+z_{2}\right)\left(Z_{33}+z_{3}\right)-Z_{23} Z_{32}}
$$

Likewise, we get similar inequality conditions for $-z_{2}$ and $-z_{3}$. So, the stability condition (9) is satisfied if and only if

$$
-z_{\kappa} \neq Z_{i n_{\kappa}}, \quad \kappa=1,2,3
$$

Now, recall that the real part of $z_{\kappa}$ covers the closed right half plane (open right half plane) if it is passive (strictly passive). Thus, the three-port network is stable (weakly stable) if and only if

$$
\operatorname{Re}\left(Z_{i n_{\kappa}}\right)>0 \quad(\geq 0), \quad \kappa=1,2,3
$$

for all passive (strictly passive) $z_{1}, z_{2}$ and $z_{3}$. 


\section{Main Result: A Stability Criterion for a Class OF NONRECIPROCAL THREE-PORT NetWorKS}

The previous stability definitions can hardly be used as closed-form stability criteria for general nonreciprocal networks. Instead, we will introduce an approach in this section that utilizes the Lemma 1 below for checking the stability of a reciprocal network, which has a symmetric impedance matrix. Also, Lemma 2 will be used for finding the symmetric equivalent of an asymmetric impedance matrix from a network stability perspective. Lastly, Lemma 3 and Lemma 4 will be required in the proof of Theorem 1 .

Lemma 1. [13] Let $Z=Z^{\mathrm{T}}$ be the impedance matrix of a reciprocal n-port network. Then, the network is passive (strictly passive), i.e., $R e Z \geq 0(\operatorname{ReZ}>0)$, if and only if it is weakly stable (stable).

Lemma 2. [16] Let $Z_{1}$ and $Z_{2}$ be the impedance matrices of two n-port networks. Then, if $Z_{1}$ and $Z_{2}$ possess identical principal minors of all orders, the two n-port networks are stable (weakly stable) together.

In fact, [16] showed that if $Z_{1}$ and $Z_{2}$ have identical principal minors of all orders, then

$$
\operatorname{det}\left(Z_{1}+Z_{0}\right)=\operatorname{det}\left(Z_{2}+Z_{0}\right)
$$

Therefore, the stability (weak stability) of the two networks with impedance matrices $Z_{1}$ and $Z_{2}$ will happen at the same time because of (2), which is to hold for all passive (strictly passive) $Z_{0}=\operatorname{diag}\left[z_{1}, z_{2}, \cdots, z_{n}\right]$.

Lemma 3. [17] A symmetric matrix is positive definite (positive semi-definite) if and only if the determinants of every principal minor is positive (nonnegative).

Lemma 4. [18] If $\operatorname{det} Z(s) \neq 0$, then $Z(s)$ is positive real if and only if $Z(s)^{-1}$ is positive real.

Now, we propose the following theorem as a compact, straightforward, and easy to check condition for the stability of a general nonreciprocal three-port network.

Theorem 1. The nonreciprocal three-port network with the impedance matrix $Z$ in (5) satisfying the symmetrization condition

$$
Z_{13} Z_{21} Z_{32}-Z_{12} Z_{23} Z_{31}=0
$$

is stable (weakly stable) if and only if

$\operatorname{Re}\left(Z_{11}\right)>0 \quad(\geq 0)$,

$\operatorname{Re}\left(Z_{22}\right)>0 \quad(\geq 0)$,

$\operatorname{Re}\left(Z_{33}\right)>0 \quad(\geq 0)$,

$\operatorname{Re}\left(Z_{11}\right) \operatorname{Re}\left(Z_{22}\right)-\frac{\left|Z_{12} Z_{21}\right|+\operatorname{Re}\left(Z_{12} Z_{21}\right)}{2}>0 \quad(\geq 0)$,

and

$$
\begin{aligned}
& \operatorname{Re}\left(Z_{11}\right) \operatorname{Re}\left(Z_{22}\right) \operatorname{Re}\left(Z_{33}\right) \\
& -\operatorname{Re}\left(Z_{11}\right) \frac{\left|Z_{23} Z_{32}\right|+\operatorname{Re}\left(Z_{23} Z_{32}\right)}{2} \\
& -\operatorname{Re}\left(Z_{22}\right) \frac{\left|Z_{13} Z_{31}\right|+\operatorname{Re}\left(Z_{13} Z_{31}\right)}{2} \\
& -\operatorname{Re}\left(Z_{33}\right) \frac{\left|Z_{12} Z_{21}\right|+\operatorname{Re}\left(Z_{12} Z_{21}\right)}{2} \\
& +2 \operatorname{Re}\left(\sqrt{Z_{12} Z_{21}}\right) \operatorname{Re}\left(\sqrt{Z_{13} Z_{31}}\right) \operatorname{Re}\left(\sqrt{Z_{23} Z_{32}}\right)>0 \quad(\geq 0)
\end{aligned}
$$

Proof. According to Lemma 2, if there exists a reciprocal threeport network with impedance matrix $Z_{e q}$ that has the same stability (weak stability) characterization as the nonreciprocal three-port network with impedance matrix $Z$, then

$$
\operatorname{det}\left(Z_{e q}+Z_{0}\right)=\operatorname{det}\left(Z+Z_{0}\right)
$$

for any passive (strictly passive) $Z_{0}=\operatorname{diag}\left[z_{1}, z_{2}, z_{3}\right]$. According to (17) in the paper, we have

$$
\begin{array}{r}
\operatorname{det}\left[\begin{array}{ccc}
Z_{a}+z_{1} & Z_{b} & Z_{d} \\
Z_{b} & Z_{c}+z_{2} & Z_{f} \\
Z_{d} & Z_{f} & Z_{h}+z_{3}
\end{array}\right] \\
=\operatorname{det}\left[\begin{array}{ccc}
Z_{11}+z_{1} & Z_{12} & Z_{13} \\
Z_{21} & Z_{22}+z_{2} & Z_{23} \\
Z_{31} & Z_{32} & Z_{33}+z_{3}
\end{array}\right]
\end{array}
$$

Calculating the two determinants and equating the coefficients of $z_{1}, z_{2}$, and $z_{3}$ (because the above is to hold for any passive (or strictly passive) $\left.Z_{0}=\operatorname{diag}\left[z_{1}, z_{2}, z_{3}\right]\right)$, if and only if the symmetrization condition (15) holds, we get

$$
Z_{e q}=\left[\begin{array}{ccc}
Z_{11} & \gamma_{1} \sqrt{Z_{12} Z_{21}} & \gamma_{2} \sqrt{Z_{13} Z_{31}} \\
\gamma_{1} \sqrt{Z_{12} Z_{21}} & Z_{22} & \gamma_{3} \sqrt{Z_{23} Z_{32}} \\
\gamma_{2} \sqrt{Z_{13} Z_{31}} & \gamma_{3} \sqrt{Z_{23} Z_{32}} & Z_{33}
\end{array}\right]
$$

where $\gamma_{i}= \pm 1$ for $i=1,2,3$. We will discuss later why the stability condition will be the same for any of these 8 solutions.

According to Lemma 1, the symmetric three-port network with the impedance matrix $Z_{e q}$ given in (18) is stable (weakly stable) if and only if it is strictly passive (passive), i.e.,

$$
\operatorname{Re}\left(Z_{e q}\right)>0 \quad(\geq 0)
$$

Consequently, if (15) holds, then the nonreciprocal three-port network with the impedance matrix $Z$ given in (5) is stable (weakly stable) if and only if the matrix $\operatorname{Re}\left(Z_{e q}\right)$, with $Z_{e q}$ given in (18), is positive definite (positive semi-definite). After simplifying the matrix $\operatorname{Re}\left(Z_{e q}\right)$ by

$$
\left(\operatorname{Re}\left(\sqrt{Z_{i j} Z_{j i}}\right)\right)=\sqrt{\frac{\left|Z_{i j} Z_{j i}\right|+\operatorname{Re}\left(Z_{i j} Z_{j i}\right)}{2}}, \quad i, j=1,2,3
$$

and using Lemma 3, we arrive at conditions (16a)-(16e) for positive definiteness (positive semi-definiteness) of $\operatorname{Re}\left(Z_{e q}\right)$. Note that any of the 8 choices caused by taking $\gamma_{i}= \pm 1, i=1,2,3$, in (18) will result in the same stability conditions (16a)-(16e) due to the fact that we are calculating the determinants of the principal minors of $Z_{e q}$. This concludes the proof.

Remark 1. Note that Theorem 1 holds not only for the impedance matrix (5) of a general nonreciprocal network but also for its admittance matrix. The reason for this is Lemma 3 and Lemma 4. Therefore, if we can show that similar conditions hold for the hybrid matrix $(H)$ representation of a three-port network, the same will hold for its inverse, which is the $G$ representation. This means the positive definiteness (positive semi-definiteness) of the equivalent reciprocal network is independent of whether an impedance representation or an immitance representation is used for it. In the following, we demonstrate that similar conditions hold for hybrid parameters of a trilateral haptic system. For a nonreciprocal three-port network given by the hybrid matrix

$$
H=\left[\begin{array}{lll}
H_{11} & H_{12} & H_{13} \\
H_{21} & H_{22} & H_{23} \\
H_{31} & H_{32} & H_{33}
\end{array}\right]
$$


the hybrid parameters are related to the impedance parameters via

$$
\begin{array}{ll}
H_{11}=\frac{1}{Z_{33}}, & H_{12}=-\frac{Z_{31}}{Z_{33}}, \quad H_{13}=-\frac{Z_{32}}{Z_{33}}, \\
H_{21}=\frac{Z_{13}}{Z_{33}}, & H_{22}=Z_{11}-\frac{Z_{13} Z_{31}}{Z_{33}}, \quad H_{23}=Z_{12}-\frac{Z_{13} Z_{32}}{Z_{33}}, \\
H_{31}=\frac{Z_{23}}{Z_{33}}, & H_{32}=Z_{21}-\frac{Z_{23} Z_{31}}{Z_{33}}, \quad H_{33}=Z_{22}-\frac{Z_{23} Z_{32}}{Z_{33}}
\end{array}
$$

Replacing these in various conditions in Theorem 1, we get the following:

$$
H_{13} H_{21} H_{32}-H_{12} H_{23} H_{31}=0
$$

and

$\begin{array}{ll}\operatorname{Re}\left(H_{11}\right)>0 & (\geq 0), \\ \operatorname{Re}\left(H_{22}\right)>0 & (\geq 0), \\ \operatorname{Re}\left(H_{33}\right)>0 & (\geq 0),\end{array}$$$
\operatorname{Re}\left(H_{11}\right) \operatorname{Re}\left(H_{22}\right)-\frac{\left|H_{12} H_{21}\right|+\operatorname{Re}\left(H_{12} Z_{21}\right)}{2}>0 \quad(\geq 0),
$$

and

$$
\begin{aligned}
& \operatorname{Re}\left(H_{11}\right) \operatorname{Re}\left(H_{22}\right) \operatorname{Re}\left(H_{33}\right) \\
& -\operatorname{Re}\left(H_{11}\right) \frac{\left|H_{23} H_{32}\right|+\operatorname{Re}\left(H_{23} Z_{32}\right)}{2} \\
& -\operatorname{Re}\left(H_{22}\right) \frac{\left|H_{13} H_{31}\right|+\operatorname{Re}\left(H_{13} Z_{31}\right)}{2} \\
& -\operatorname{Re}\left(H_{33}\right) \frac{\left|H_{12} H_{21}\right|+\operatorname{Re}\left(H_{12} Z_{21}\right)}{2} \\
& +2 \operatorname{Re}\left(\sqrt{H_{12} H_{21}}\right) \operatorname{Re}\left(\sqrt{H_{13} H_{31}}\right) \operatorname{Re}\left(\sqrt{H_{23} H_{32}}\right)>0 \quad(\geq 0)
\end{aligned}
$$

Obviously, conditions (22) and (23) are the same as those in Theorem 1 with the difference that hybrid parameters have replaced impedance parameters.

Remark 2. For the special case of $Z_{13}=Z_{23}=Z_{31}=Z_{32}=$ $Z_{33}=0$, Theorem 1 simplifies to the stability criterion for nonreciprocal two-port networks best known as Llewellyn's criterion $[1,2,3]$. Also, for the special case of $Z_{13}=Z_{31}=0$, Theorem 1 simplifies to the stability criterion in [12]. Our Theorem 1 is more general as it lifts those constraints and is applicable to any nonreciprocal three-port network whose impedance matrix $Z$ satisfies the symmetrization condition (15). As we will demonstrate in the next section, the symmetrization condition (15) is mild and can be fulfilled by appropriate choice of free parameters in the three-port network (e.g., controller structure and gains in the case of trilateral haptic systems).

\section{Case Study: Application of the Proposed Crite- RION TO A TRILATERAL HAPTIC SYSTEM}

In this section, the aim is to apply the proposed stability criterion, which is general and can be used for three-port networks in various applications, to a trilateral haptic system. A trilateral haptic system may be a collaborative haptic virtual environment with three users, or a dual-user haptic teleoperation system with one slave robot. In the following, for brevity, we only consider the former and a similar procedure case may be followed for the latter.

\subsection{A triple-user collaborative haptic virtual environ- ment system}

In one degree of freedom triple-user collaborative haptic virtual environment systems, the goal is that three users cooperate with one another in a virtual environment to perform a task while receiving haptic feedback. This corresponds to multi-point-of-contact interaction with a virtual environment [19]. The system consists of three

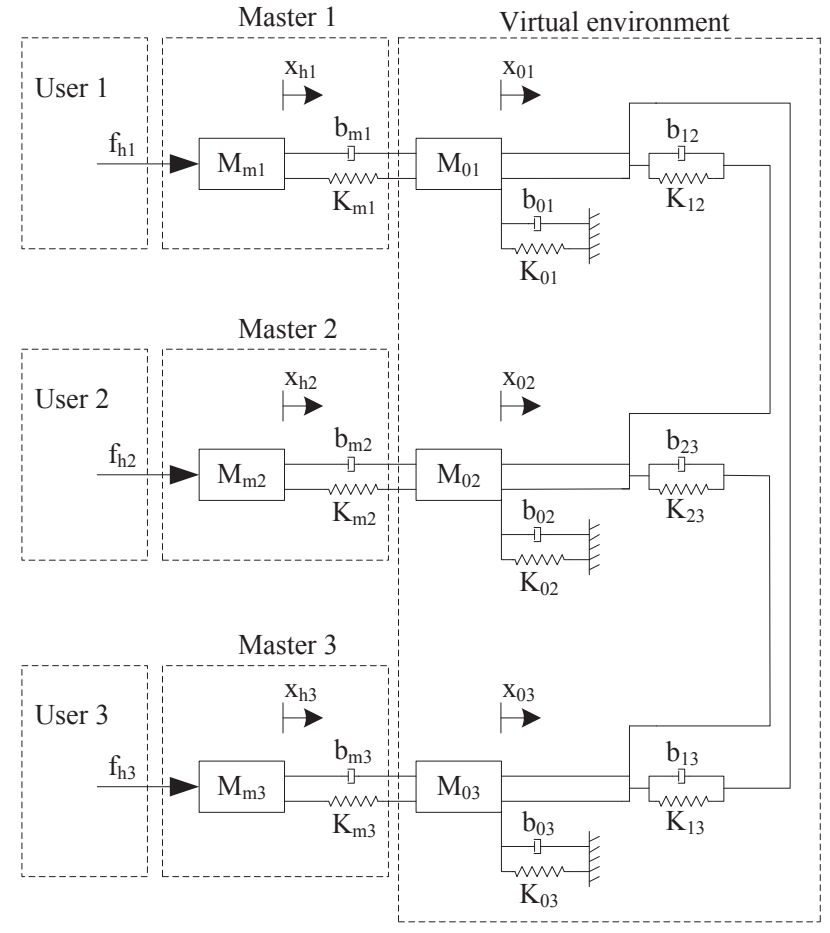

Figure 2: A triple-user collaborative haptic virtual environment system.

master robots, each of which operating on a specific point on the virtual object as shown in Figure 2. The virtual object computes the dynamic response (in terms of force feedback) at each of these points by using the positions of the three master user interfaces. One application of such a trilateral system is in tele-rehabilitation, in which two master robots are operated by two patients and the third master robot is operated by a therapist. The therapist interacts with the patients in a virtual environment designed for rehabilitation exercises and monitoring their progress through the received force (and visual) feedback.

Virtual object's mechanical properties such as mass, stiffness, and damping can be adjusted to correspond to real life objects. Dynamic modeling of the virtual environment based on a mass-springdamper mesh follows. Consider the triple-user collaborative haptic virtual environment system shown in Figure 2. In this figure, $M_{m i}$, $K_{m i}$ and $b_{m i}, i=1,2,3$, are mass, stiffness, and damping terms of the three masters. Also, $M_{0 i}$ represents the mass of a node of the virtual object mesh that is in contact with master $i$. We assume $M_{0 i}$ is connected to a stationary ground via spring $K_{0 i}$ and damper $b_{0 i}$. We also assume $K_{12}, K_{13}, K_{23}$ are the stiffness of springs connecting the three nodes of the mesh of the virtual object. Similarly, $b_{12}$, $b_{13}, b_{23}$ are the dampers connecting the same three nodes. Lastly, $f_{h i}$ denotes the interaction force between each user and the corresponding master.

The dynamics of the three masters are

$$
\left\{\begin{array}{l}
M_{m 1} \ddot{x}_{h 1}=f_{h 1}+K_{m 1}\left(x_{01}-x_{h 1}\right)+b_{m 1}\left(\dot{x}_{01}-\dot{x}_{h 1}\right) \\
M_{m 2} \ddot{x}_{h 2}=f_{h 2}+K_{m 2}\left(x_{02}-x_{h 2}\right)+b_{m 2}\left(\dot{x}_{02}-\dot{x}_{h 2}\right) \\
M_{m 3} \ddot{x}_{h 3}=f_{h 3}+K_{m 3}\left(x_{03}-x_{h 3}\right)+b_{m 3}\left(\dot{x}_{03}-\dot{x}_{h 3}\right)
\end{array}\right.
$$

Also, the dynamics of the three nodes on the mesh of the virtual 
object are

$$
\left\{\begin{aligned}
M_{01} \ddot{x}_{01} & =K_{m 1}\left(x_{h 1}-x_{01}\right)+b_{m 1}\left(\dot{x}_{h 1}-\dot{x}_{01}\right) \\
& +K_{12}\left(x_{02}-x_{01}\right)+b_{12}\left(\dot{x}_{02}-\dot{x}_{01}\right) \\
& +K_{13}\left(x_{03}-x_{01}\right)+b_{13}\left(\dot{x}_{03}-\dot{x}_{01}\right) \\
& +K_{01}\left(0-x_{01}\right)+b_{01}\left(0-\dot{x}_{01}\right) \\
M_{02} \ddot{x}_{02} & =K_{m 2}\left(x_{h 2}-x_{02}\right)+b_{m 2}\left(\dot{x}_{h 2}-\dot{x}_{02}\right) \\
& +K_{12}\left(x_{01}-x_{02}\right)+b_{12}\left(\dot{x}_{01}-\dot{x}_{02}\right) \\
& +K_{23}\left(x_{03}-x_{02}\right)+b_{23}\left(\dot{x}_{03}-\dot{x}_{02}\right) \\
& +K_{02}\left(0-x_{02}\right)+b_{02}\left(0-\dot{x}_{02}\right) \\
M_{03} \ddot{x}_{03} & =K_{m 3}\left(x_{h 3}-x_{03}\right)+b_{m 3}\left(\dot{x}_{h 3}-\dot{x}_{03}\right) \\
& +K_{13}\left(x_{01}-x_{03}\right)+b_{13}\left(\dot{x}_{01}-\dot{x}_{03}\right) \\
& +K_{23}\left(x_{02}-x_{03}\right)+b_{23}\left(\dot{x}_{02}-\dot{x}_{03}\right) \\
& +K_{03}\left(0-x_{03}\right)+b_{03}\left(0-\dot{x}_{03}\right)
\end{aligned}\right.
$$

For simplicity, let us choose $b_{m 1}=b_{m 2}=b_{m 3}=b_{01}=b_{02}=b_{03}=$ $b_{12}=b_{13}=b_{23}=0$. Thus, the impedance matrix representation of the closed-loop triple-user haptic virtual environment system is

$$
\left[\begin{array}{l}
f_{h 1} \\
f_{h 2} \\
f_{h 3}
\end{array}\right]=\left[\begin{array}{lll}
Z_{11} & Z_{12} & Z_{13} \\
Z_{21} & Z_{22} & Z_{23} \\
Z_{31} & Z_{32} & Z_{33}
\end{array}\right]\left[\begin{array}{c}
\dot{x}_{h 1} \\
\dot{x}_{h 2} \\
\dot{x}_{h 3}
\end{array}\right]
$$

where $Z=A^{-1} B$ with

$$
\begin{gathered}
A=\left[\begin{array}{ccc}
a_{1} s & -\frac{K_{12}}{K_{m 2}} & -\frac{K_{13}}{K_{m 3}} \\
-\frac{K_{12}}{K_{m 1}} & a_{2} s & -\frac{K_{23}}{K_{m 3}} \\
-\frac{K_{13}}{K_{m 1}} & -\frac{K_{23}}{K_{m 2}} & a_{3} s
\end{array}\right] \\
B=\left[\begin{array}{ccc}
\frac{a_{1} q_{1} s-K_{m 1}}{s} & -\frac{q_{2} K_{12}}{s} & -\frac{q_{3} K_{13}}{s} \\
-\frac{q_{1} K_{12}}{s} & \frac{a_{2} q_{2} s-K_{m 2}}{s} & -\frac{q_{3} K_{23}}{s} \\
-\frac{q_{1} K_{13}}{s} & -\frac{q_{2} K_{23}}{s} & \frac{a_{3} q_{3} s-K_{m 3}}{s}
\end{array}\right]
\end{gathered}
$$

In the above, $a_{1}=M_{01} s+\frac{K_{m 1}+K_{12}+K_{13}+K_{01}}{s}, a_{2}=M_{02} s+$ $\frac{K_{m 2}+K_{12}+k_{23}+K_{02}}{s}, a_{3}=M_{03} s+\frac{K_{m 3}+K_{13}+K_{23}+K_{03}}{s}, q_{1}=\frac{M_{m 1} s^{2}+K_{m 1}}{K_{m 1}}$, $q_{2}=\frac{M_{m 2} s^{2}+K_{m 2}}{K_{m 2}}$, and $q_{3}=\frac{M_{m 3} s^{2}+K_{m 3}}{K_{m 3}}$.

\subsection{Stability analysis}

It can be shown that the symmetrization condition (15) will always hold once we find the elements of the three-port network impedance matrix (26). It is easy to see that the left side of stability conditions (16a)-(16c) will always equal zero. Condition (16d) turns out to be

$$
\frac{1}{2} Q_{1}^{2} Q_{2} Q_{3}\left(-1+\operatorname{sign}\left(Q_{2}\right) \operatorname{sign}\left(Q_{3}\right)\right) \operatorname{sign}\left(Q_{2}\right) \operatorname{sign}\left(Q_{3}\right) \geq 0
$$

where

$$
\begin{aligned}
& Q_{1}=K_{12} K_{m 3} M_{03} \omega^{2}-K_{12} K_{m 3} K_{23}-K_{12} K_{m 3} K_{13}-K_{12} K_{m 3} K_{03} \\
& -K_{23} K_{13}-K_{12} K_{m 3}^{2} \\
& Q_{2}=\omega^{4} M_{m 1} M_{01}\left(K_{m 1}-1\right)-\omega^{2}\left(K_{m 1}-1\right)\left(M_{m 1} K_{m 1}+K_{m 1} K_{01}\right. \\
& \left.+K_{m 1} M_{m 1}+K_{12} M_{m 1}+K_{13} M_{m 1}\right)+K_{m 1}\left(K_{m 1}-1\right)\left(K_{12}+K_{13}+K_{01}\right) \\
& +K_{m 1}^{3} \\
& Q_{3}=\omega^{4} M_{m 2} M_{02}\left(K_{m 2}-1\right)-\omega^{2}\left(K_{m 2}-1\right)\left(M_{m 2} K_{m 2}+K_{m 2} K_{02}\right. \\
& \left.+K_{m 2} M_{m 2}+K_{12} M_{m 2}+K_{23} M_{m 2}\right)+K_{m 2}\left(K_{m 2}-1\right)\left(K_{12}+K_{23}+K_{02}\right) \\
& +K_{m 2}^{3}
\end{aligned}
$$

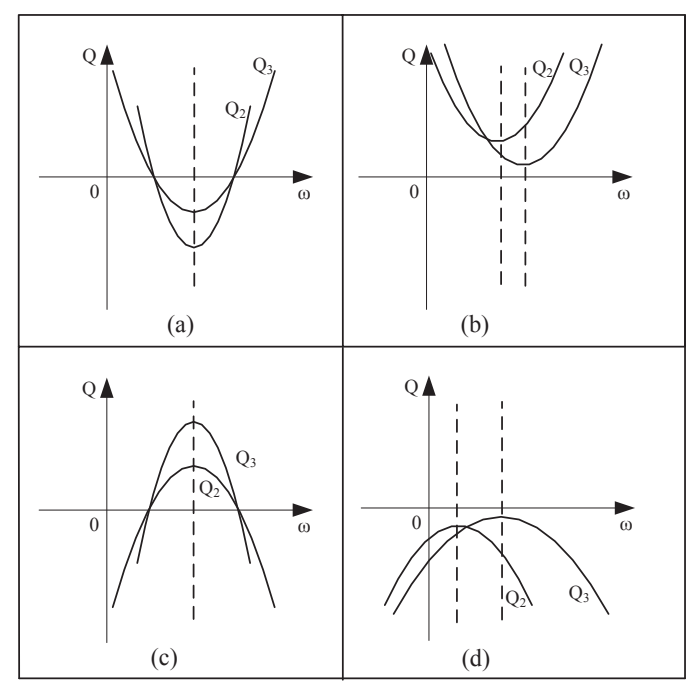

Figure 3: The four cases when $Q_{2}$ and $Q_{3}$ have the same sign.

Obviously, (27) will be fulfilled for all frequencies $\omega$ if $K_{m 1}=$ $K_{m 2}=1$. Also, if $Q_{2}$ and $Q_{3}$ have the same sign, (27) will be fulfilled.

Given that $Q_{2}$ and $Q_{3}$ are quadratic polynomials in $\omega$, there is a total of 4 possibilities as shown in Figure 3 for the signs of $Q_{2}$ and $Q_{3}$. Here, we only consider the case (b). In this case, a sufficient condition for stability is

$$
\begin{aligned}
& K_{m 1}>1, \quad K_{m 2}>1, \quad M_{m 1}>M_{o 1}, \quad M_{m 2}>M_{02} \\
& 4 K_{m 1}^{3}>\left(K_{m 1}-1\right)\left(K_{12}+K_{13}+K_{01}\right)^{2} \\
& 4 K_{m 2}^{3}>\left(K_{m 2}-1\right)\left(K_{12}+K_{23}+K_{02}\right)^{2}
\end{aligned}
$$

These conditions will make the left hand side of (27) equal to zero. Since the left side of conditions (16a)-(16d) have become identical to zero, so, the left side of condition (16e) will also equal zero, and stability is ensured.

Given the symmetry between the three ports in a trilateral system, a sufficient, frequency-independent, and compact condition for stability of the above-described triple-user collaborative haptic virtual environment system is either

$$
K_{m 1}=K_{m 2}=K_{m 3}=1
$$

or

$$
\left\{\begin{array}{l}
K_{m 1}>1, \quad M_{m 1}>M_{01}, \\
K_{m 2}>1, \quad M_{m 2}>M_{02}, \\
K_{m 3}>1, \quad M_{m 3}>M_{03}, \\
4 K_{m 1}^{3}>\left(K_{m 1}-1\right)\left(K_{12}+K_{13}+K_{01}\right)^{2}, \\
4 K_{m 2}^{3}>\left(K_{m 2}-1\right)\left(K_{12}+K_{23}+K_{02}\right)^{2}, \\
4 K_{m 3}^{3}>\left(K_{m 3}-1\right)\left(K_{13}+K_{23}+K_{03}\right)^{2} .
\end{array}\right.
$$

\section{Simulations}

In this section, the stability condition for the triple-user collaborative haptic virtual environment system found in the previous section will be verified via simulations. For checking the stability of the triple-user collaborative haptic virtual environment system, the ports \#2 and \#3 were connected to passive terminations while the 
Table 1: The controller gains of the triple-user collaborative haptic virtual environment system used in simulations. (A) Stable, (B) Potentially unstable.

\begin{tabular}{l|ll|ll|ll|ll}
\hline & \multicolumn{10}{|c}{ Parameters } \\
\hline (A) & $M_{01}$ & 0.4 & $K_{m 1}$ & 260 & $K_{01}$ & 15 & $K_{12}$ & 6 \\
& $M_{02}$ & 0.4 & $K_{m 2}$ & 260 & $K_{02}$ & 15 & $K_{23}$ & 6 \\
& $M_{03}$ & 0.4 & $K_{m 3}$ & 260 & $K_{03}$ & 15 & $K_{13}$ & 6 \\
\hline (B) & $M_{01}$ & 0.4 & $K_{m 1}$ & 2 & $K_{01}$ & 6 & $K_{12}$ & 3 \\
& $M_{02}$ & 0.4 & $K_{m 2}$ & 1 & $K_{02}$ & 6 & $K_{23}$ & 3 \\
& $M_{03}$ & 0.4 & $K_{m 3}$ & 3 & $K_{03}$ & 6 & $K_{13}$ & 3 \\
\hline
\end{tabular}

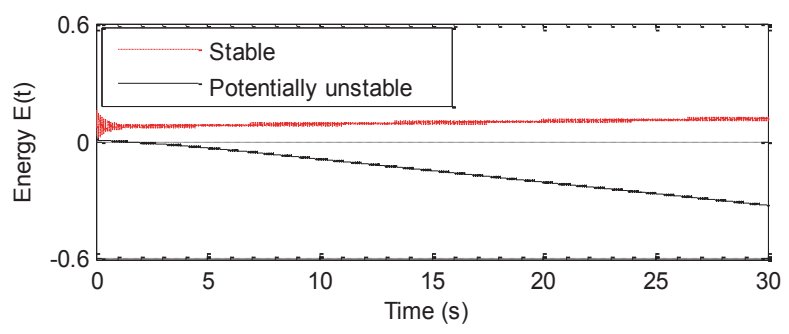

Figure 4: (a) Input energy at the \#1's port of a triple-user collaborative haptic virtual environment system. Simulation parameters are listed in Table I: parameters (A) for the stable, parameters $(B)$ for the potentially unstable.

input energy at the port \#1 (i.e., the energy dissipation in the threeport network terminated in ports 2 and 3 ) was measured. The system is absolutely stable if and only if, at all times $t>0$, we have [15]:

$$
E(t)=\int_{0}^{t} f_{h 1}(\tau) \dot{x}_{h 1}(\tau) \mathrm{d} \tau>0 \quad(\geq 0)
$$

The triple-user collaborative haptic virtual environment system has been simulated in MATLAB/Simulink. There is no time delay in the communication channel between the masters and the virtual objects. Three 1-DOF robots as the three masters are modeled by masses $M_{m 1}=M_{m 2}=M_{m 3}=1.6$, respectively. The master \#2 and \#3 are connected to LTI terminations with transfer functions $\frac{1}{s+1}$, which are strictly passive as, for $s=j \omega$, we have $\operatorname{Re}\left(\frac{1}{s+1}\right)=\frac{1}{\omega^{2}+1}>0$ when $\omega>0$. A sine-wave input $f_{h 1}$ is applied to the master \#1's open port.

According to (29) and (30), the stability of the triple-user haptic system should depend on the controller gains and robots parameters. In the simulations, the parameters $M_{m i}, M_{0 i}, K_{m i}, K_{01}, K_{12}$, $K_{13}$, and $K_{23}$, where $i=1,2,3$, were chosen according to Table 1 . The input energy (31) profiles are plotted in Figure 4(a). As it can be seen, if the controller gains are selected according to (30), e.g., as listed in Table 1(A), then the input energy at port 1 is positive at all times, indicating the stability of the trilateral haptic system. However, when the controller gains violate (29) or (30), e.g., as listed in Table 1(B), the input energy may become negative at least for a period of time, indicating potential instability of the trilateral system. We get similar results if we repeat the above simulations after replacing the strictly passive terminations $\frac{1}{s+1}$ by the passive terminations $\frac{1}{s}$, and do not report its results for brevity. These results validate the previously-found stability conditions.

\section{Conclusions}

We presented a closed-form stability criterion for a three-port network based on its immitance matrix. While the proposed criterion (Theorem 1) can be used for stability analysis of a general class of three-port networks in a variety of applications, we elaborated on its application in stability analysis of trilateral haptic systems. Through simulations involving a triple-user collaborative haptic virtual environment system, the proposed analytical stability criterion was validated.

\section{REFERENCES}

[1] F. Llewellyn, "Some fundamental properties of transmission systems," Proceedings of the IRE, vol. 2, no. 1, pp. 271-283, 1952.

[2] W. Ku, "A simple dervation for the stability criterion of linear active two-port," Proceedings of the IEEE, pp. 310 - 311, March 1965.

[3] S. Haykin, Active Network Theory. Reading, MA: Addison-Wesley, 1970.

[4] C. R. Carignan and P. A. Olsson, "Cooperative control of virtual objects over the internet using force-reflecting master arms," in Proc. of IEEE Int. Conf. on Rob. And Auto., vol. 2, pp. 1221-1226, 2004.

[5] S. Nudehi, R. Mukherjee, and M. Ghodoussi, "A shared-control approach to haptic interface design for minimally invasive telesurgical training," IEEE Transactions on Control Systems Technology, vol. 13, no. 4, pp. 588-592, July 2005.

[6] W. T. Lo, Y. Liu, I. Elhajj, N. Xi, Y. Wang, and T. Fukuda, "Cooperative teleoperation of a multirobot system with force reflection via internet," IEEE/ASME Transactions on Mechatronics, vol. 9, no. 4, pp. $661-670$, December 2004.

[7] R. F. Kuo and T. H. Chu, "Unconditional stability boundaries of a three-port network," IEEE TMTTechniques, vol. 58, no. 2, pp. 363371, December 2010.

[8] E. Tan, "Simplified graphical analysis of linear three-port stability," IEE Proceedings on Microwaves, Antennas and Propagation, vol. 152, no. 4, pp. 209-213, August 2005.

[9] B. Khademian and K. Hashtrudi-Zaad, "Unconditional stability analysis of dual-user teleoperation systems," in 2010 IEEE Haptics Symposium, March 2010, pp. $161-166$.

[10] J. Boehm and W. Albright, "Unconditional stability of a three-port network characterized with s-parameters," IEEE Transactions on Microwave Theory and Techniques, vol. 35, no. 6, pp. 582 - 586, January 1987.

[11] J. Choma and W. K. Chen, Feedback Networks: Theory and Circuit Applications. World Scientific Publishing Company, 2007.

[12] W. Ku, "Stability of linear active nonreciprocal n-ports," J. Franklin Inst., no. 276, pp. 207 - 224, 1963.

[13] D. Youla, "A stability characterization of the reciprocal linear passive n-port," Proc. IRE, vol. 47, pp. 1150-1151, 1959.

[14] S. Boyd and O. Chua, "On the passivity criterion for LTI n-port," Circuit theory and applications, vol. 10, pp. 323-333, 1982.

[15] H. J. Marquez, Nonlinear Control Systems Analysis and Design. Wiley, 2003.

[16] D. Youla, "A note on the stability of linear, nonreciprocal n-port," Proc. IRE, vol. 48, pp. 121-122, 1960.

[17] H. Anton and C. Rorres, Elementray linear algebra: Applications version. Wiley, 2005, vol. 9.

[18] W. Enping and W. Chaozhu, "Some properties of the positive real matrix," Acta Mathematicae applicatae sinica, vol. 1, no. 1, pp. 271$283,1984$.

[19] D. Lee and K. Huang, "Peer-to-peer control architecture for multiuser haptic collaboration over undirected delayed packet-switching network," 2010 IEEE International Conference on Robotics and Automation, pp. 1333-1338, May 2010. 